\title{
Docencia en la unAm desde la mirada de los rectores
}

\author{
Tania Itzel Nieto Juárez
}

\begin{abstract}
Resumen
¿Alguna vez te has preguntado cuál es la labor que han tenido y tienen encomendada las maestras y los maestros de nuestra Universidad? Para contestar la pregunta, en este artículo, en primer lugar, se realiza una breve introducción en torno a la función docente en las universidades del siglo XXI: la concepción y los elementos que ésta debería poseer; en segundo lugar, se hace alusión a la tarea docente en la Universidad Nacional Autónoma de México (UNAM) y, finalmente, se da a conocer, a través de los discursos de su fundador y algunos de sus rectores, la idea que éstos han tenido respecto a la docencia y la labor que asignan o reconocen en las y los docentes unamitas, en determinado tiempo y espacio. Pero ¿por qué se considera pertinente recuperar el pensamiento de los rectores? Porque son ellos quienes, con base en las múltiples expresiones de la comunidad universitaria, determinan las acciones que realizará la Universidad durante su gestión, en este caso, en materia de docencia, de acuerdo con las condiciones institucionales y contextuales.
\end{abstract}

Palabras clave: universidad, docencia, maestras, maestros, rectores, UNAM.

Teaching at unam from the Perspective of the UnIVERsity's Presidents

\begin{abstract}
Have you ever wondered what is the work that the teachers of our University have had and are entrusted with? To answer this question, in this article, firstly, we review briefly the teaching function of the universities in the 21st century: the conception and the elements that it should have; secondly, we discuss the teaching task of the National Autonomous University of Mexico (UNAM), and finally, through the speeches of its founder and some of its college presidents, we examine the ideas that they have had in regard to the teaching and the work that they assign or recognize in the unamitas teachers, in a particular time and space. But why is it pertinent to recover the chancellor's perspectives? Because they are the ones who, based on the multiple expressions of the university community, determine the actions that the University will carry out during their administration, in this case, in the matter of teaching, according to institutional and contextual conditions.
\end{abstract}

Keywords: university, teaching, teachers, college presidents, chancellors, UNAM.

DOI: http://doi.org/10.22201/codeic.16076079e.2019.v20n3.a10 


\section{Tania Itzel Nieto Juárez}

Licenciada en Pedagogía por la Universidad Nacional Autónoma de México (UNAM) y especialista en entornos virtuales de aprendizaje por la Organización de Estados Iberoamericanos para la Educación, la Ciencia y la Cultura. Actualmente, es profesora de asignatura en el Colegio de Pedagogía de la Facultad de Filosofía y Letras, y estudia el tercer semestre de la Maestría en Pedagogía en la UNAM, con orientación en docencia. Realizó un semestre de movilidad estudiantil en la Universidad de Barcelona (UB) en la Facultad de Pedagogía y es alumni de la red de estudiantes del Observatori de I'Estudiant de la UB. Sus temas de interés son educación superior, prácticas y formación docente, historia de la educación y la pedagogía en México, e historia de la Universidad Nacional Autónoma de México.

"Aspirar a ser catedrático universitario no es sólo aspirar a un honor, sino a la obligación de educar, que significa, en lo más profundo, prepararse para ayudar a otros seres a formarse como hombres [seres huma-

nos],

como servidores de los demás, en la más alta categoría, a orientar para transitar por los difíciles senderos de la verdad; y a inspirar propulsando la creatividad excelsa [...]"

Héctor Solís Quiroga

\section{La docencia en las universidades}

En lo que va del siglo xxl, la institución universitaria se ha enfrentado a un escenario en el que las demandas sociales, políticas, económicas y culturales le exigen replantear constantemente sus fines y alcances, situación que la coloca en un escenario complejo por la diversidad de factores a considerar en el cumplimiento de su misión, la cual realiza incorporando elementos innovadores, sin perder de vista los elementos tradicionales que la han conservado a lo largo de su historia y que, claramente, la diferencian de otras instituciones sociales. En esta tarea, se ve imbricada toda la comunidad educativa: docentes, estudiantes, administrativos y autoridades, quienes intervienen a través de actividades específicas.

Las y los profesores son los responsables principales de la función docente en las universidades, y son quienes se enfrentan a una serie de retos que les plantea el desempeño de su tarea, tanto en el aula como en el plano institucional y social. Y es que la docencia es una tarea intencional y colaborativa que trasciende lo que sucede en los espacios universitarios, lo mismo en su ejecución que en sus alcances, los cuales se hacen evidentes en diferentes niveles y espacios de lo social. 
Imagen 1. IISUE/ AHUNAM/ Colección Universidad/ Vida Cotidiana/ CU-004649.
Sin embargo, en los últimos tiempos se han agudizado los cuestionamientos a la figura y labor del docente universitario frente a las diversas modalidades educativas y a la gran cantidad de información a la cual tienen acceso los estudiantes. Tomando en cuenta lo anterior, ¿se han preguntado qué es lo que hace un "buen profesor" para ser considerado como tal? De acuerdo con Ken Bain, en su texto Lo que hacen los mejores profesores universitarios, aquellos que pueden calificarse así son "esas personas que tienen mucho éxito a la hora de ayudar a sus estudiantes a conseguir resultados de aprendizaje extraordinarios" (Bain, 2007).

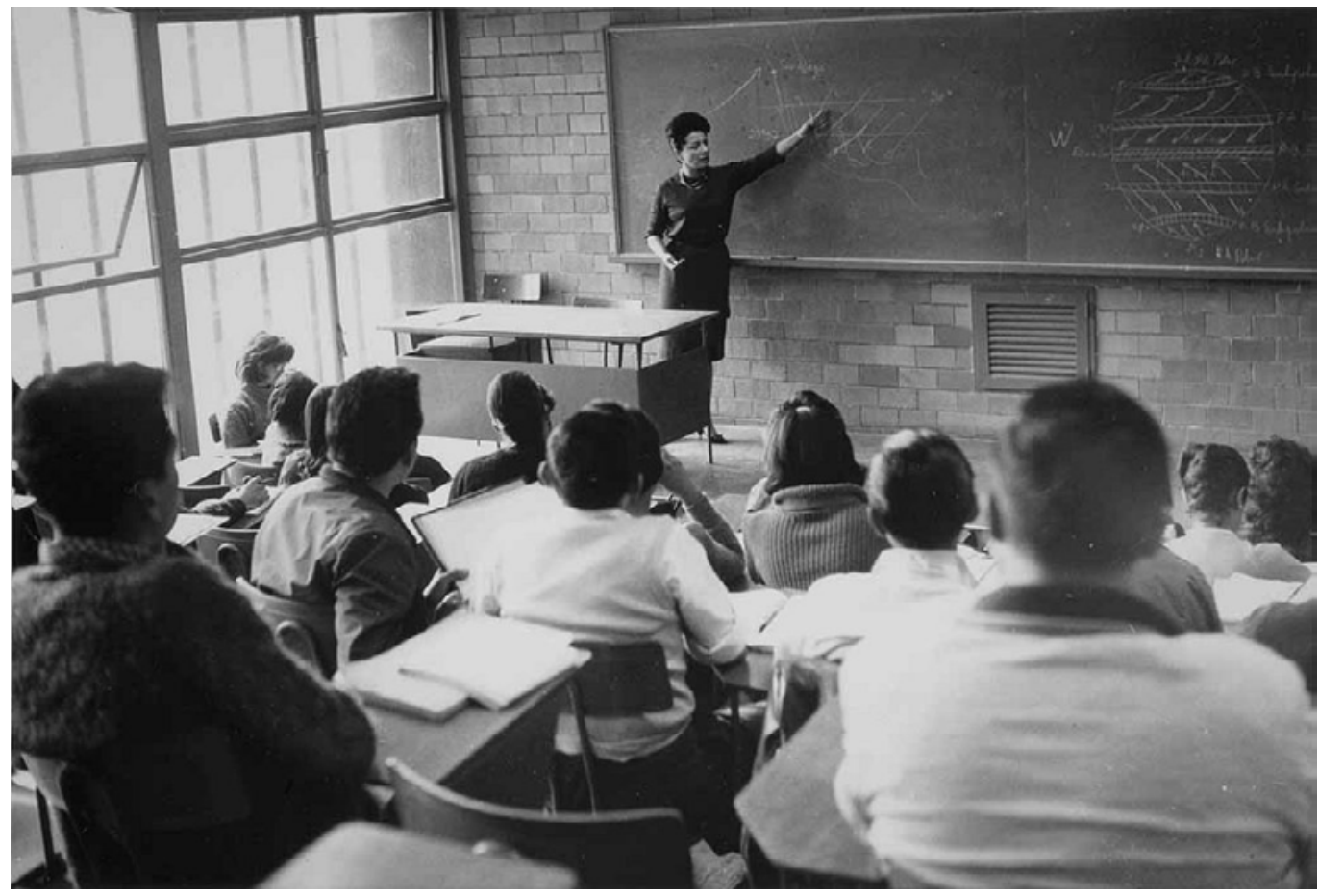

¿Pero cómo conseguir esos resultados? A decir de Francesc Imbernon (2009), el profesorado universitario que asume la importante tarea de enseñar requiere al menos de:

- Tener un dominio de la materia o disciplina que ha de impartirse. No se puede enseñar si no se sabe qué se ha de enseñar.

- Tener cierto conocimiento y habilidades para comunicarse con las personas. La comunicación es la base de la enseñanza.

- Conocer al grupo de alumnos y alumnas. Si pretendemos sintonizar, cuanto más conozcamos al grupo, mucho mejor.

- Conocer y experimentar técnicas de dinámica de grupos con diferente finalidad.

- Saber elaborar un guion de la sesión.

- Tener preparado un sistema para evaluar tanto al alumnado como su propia intervención (reflexión sobre la acción). 
Aunado a ello, las maestras y maestros universitarios dirigen tesis, participan en comisiones académicas de diversa índole y en proyectos de innovación educativa, desarrollan materiales educativos, asisten a actividades de formación y profesionalización, dan tutorías, entre otras labores.

Así, es posible afirmar que la tarea que tiene como principal objetivo la formación de profesionistas y ciudadanos responsables, reflexivos y críticos, que demanda el avance del conocimiento y la sociedad, va más allá de la mera transmisión de conocimientos, requiere de una implicación profunda con la labor académica, la cual supone crear:

[...] lo que podríamos llamar un entorno para el aprendizaje crítico natural, en el que incluyen destrezas y la información que ellos quieren enseñar mediante trabajos (preguntas y tareas) que los estudiantes encontrarán fascinantes -auténticas tareas que les provocarán curiosidad, que les motivarán a repensar sus supuestos y a examinar sus modelos mentales de la realidad-. Estos profesores crean un entorno seguro en el que los estudiantes pueden probar, quedarse cortos, realimentarse y volver a intentarlo. Los estudiantes entienden y recuerdan lo que han aprendido porque dominan y utilizan las destrezas del razonamiento necesarias para integrarlo con conceptos más amplios. Se hacen conscientes de las implicaciones y aplicaciones de las ideas y la información. Reconocen la importancia de medir su propio trabajo intelectual conforme va teniendo lugar, y durante el proceso aplican rutinariamente los estándares intelectuales de distintas disciplinas (Bain, 2007).

\section{La docencia en la Universidad Nacional Autónoma de México}

Una consideración que hay que tener presente al pensar la actividad docente es que se trata de una actividad situada; es decir, que su análisis y ejecución implica considerar las expresiones del contexto que, de maneras específicas, se manifiestan e influyen en ésta. La UNAM, a lo largo de su devenir, ha logrado posicionarse como el proyecto educativo, científico y cultural más grande del país, siendo considerada la institución educativa referente del nivel medio superior y superior a nivel nacional.

En esta institución, atendiendo al tema que aquí nos ocupa, la docencia es concebida como aquella que:

[...] debe estar vinculada con las inquietudes y problemas de la sociedad en donde se desarrolla. Nuestra Universidad debe instruir, educar y formar individuos que sirvan al país. Pretendemos preparar alumnos competentes e informados, dotados de sentido social y conciencia nacional, que actúen en convicción y sin egoísmo, que pretendan un futuro mejor en lo individual y en lo colectivo. Esto sólo se logrará en un ambiente de libertad, sin prejuicios, dogmas o hegemonías ideológicas (UNAM, 2003). 
Imagen 2. IISUE/ AHUNAM/ Colección Raúl Estrada Discua/ Otros Autores/ RED-07893.
1 La selección de los documentos y discursos de diversas personalidades, entre ellas diversos rectores, responde a momentos cruciales en la historia de la Universidad Nacional; en primer lugar se ubica a Justo Sierra, quien si bien no fue rector de la institución, fue el fundador de la misma; en segundo lugar, se recupera a José Vasconcelos, por ser el rector que, siguiendo el ideario revolucionario, dio identidad a la Universidad acuñando el escudo y lema que hasta la fecha ostenta la institución; posteriormente, se recuperan las ideas del primer rector que dirigió la institución tras la promulgación de

la autonomía universitaria, el doctor Ignacio García Téllez (1929-1932),

así como las de su sucesor, Roberto Medellín Ostos (1932-1933), en

cuya gestión la Universidad recibió la autonomía plena por parte del gobierno federal. Después se refiere el pensamiento de Alfonso Caso

(1944-1945), en cuyo rectorado se aprobó una nueva Ley Orgánica

(1945), comenzando así una nueva etapa institucional, respecto al replanteamiento de los fines y

tareas universitarias; en este mismo sentido se alude a las palabras de los rectores Genaro Fernández

MacGregor (1945-1946) y Salvador Zubirán Anchondo (1946-1948).

Finalmente, se hace un salto hasta la década de los setenta, por ser considerada como aquella en la que

da inicio la historia contemporánea de nuestra Universidad, que se extiende hasta nuestros días.
En consecuencia, es pertinente mencionar que la UNAM no tiene un único modelo educativo que sustente sus múltiples y complejas tareas académicas, más bien, existe un perfil docente diferenciado para cada nivel educativo y área de conocimiento, debido, entre otros factores, a que en esta Universidad por su carácter autónomo se hace efectivo el principio de la libertad de cátedra.

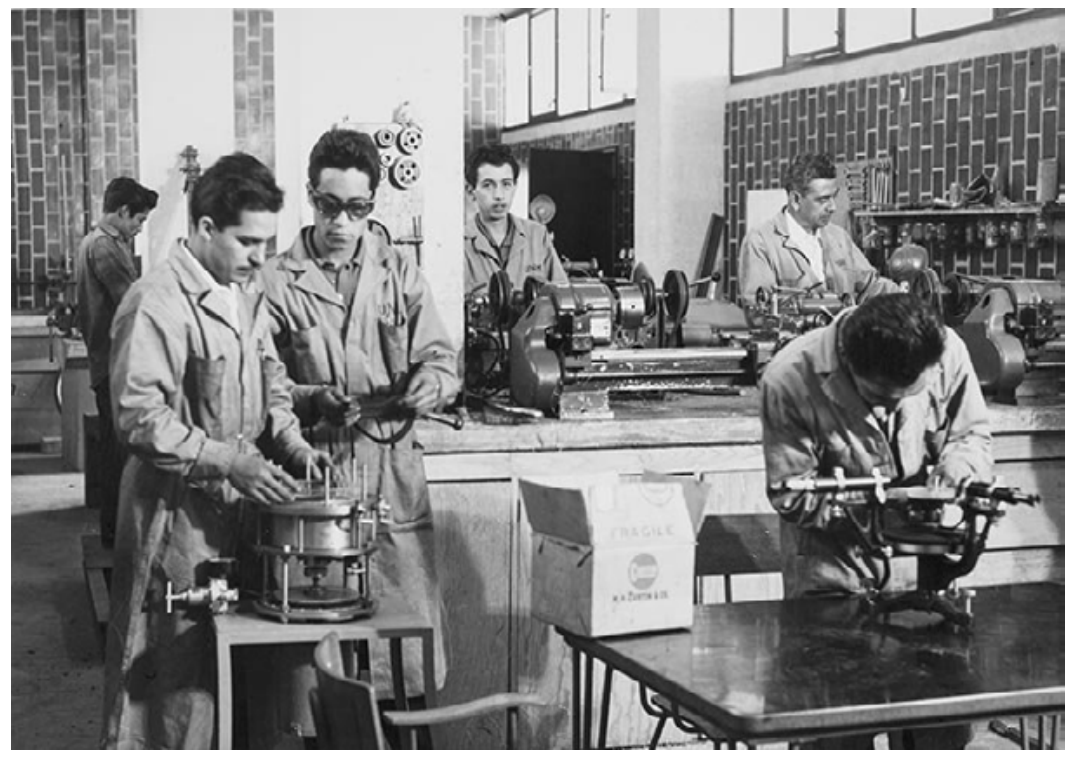

De acuerdo con lo anterior, las maestras y maestros universitarios, que gozan en nuestra Universidad de plena libertad de cátedra para impartir sus clases, deben ser conscientes de su condición como individuos y profesionales, pero también como docentes universitarios que forman parte de una institución específica que tiene a su vez un contexto, identidad, normatividad y condiciones de acción, en los que el trabajo colaborativo y el reconocimiento de su labor se ubican como factor primordial para lograr los fines asignados a la Universidad Nacional.

En este sentido, ¿alguna vez te has preguntado cómo conciben, o han concebido, las autoridades universitarias -en el caso de este artículo los rectores-, a lo largo del tiempo, la labor docente y hacia quiénes la desarrollan? La pregunta resulta relevante ya que de dicha concepción dependerá en gran medida la atención y recursos de diversa índole que se le destinen a esta tarea esencial de la institución.

\section{Discursos y documentos en los que se hace referencia a la docencia y al papel de las maestras y maestros de la UNAM $^{1}$}

A lo largo de la historia de la unAm, los rectores han manifestado cómo entienden la tarea docente, la manera en la que ésta debe desarrollarse, el impacto que las profesoras y los profesores tienen en sus estudiantes, y, de manera más 
amplia, la importancia que reviste la formación universitaria en el desarrollo del país, entre otras cuestiones. A continuación, se recuperan las palabras de algunos educadores y rectores que han hecho referencia a la tarea docente y a los ejecutores de ésta: las maestras y los maestros universitarios.

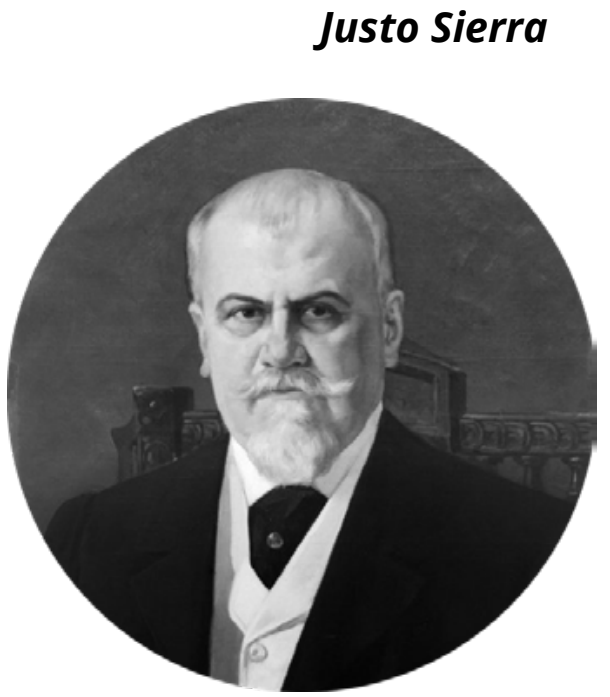

Fue el fundador de la Universidad Nacional de México. Desde la presentación de su primer proyecto de creación de una universidad mexicana, en 1881, mencionó que la instrucción es una tarea en la que se requiere esencialmente de dos actores que realmente estén involucrados en ella: el alumno y el docente. Sierra aludía a que no bastaba con que éste último dominara su disciplina, sino que era necesario que conociera el método a través del cual transmitiría los conocimientos propios de la misma (Sierra, 1881).

En su discurso pronunciado con motivo de la inauguración de la Universidad Nacional de México, el 22 de septiembre de 1910, mencionó que se imaginaba a la institución como:

Un grupo de estudiantes de todas las edades sumadas en una sola, la edad de la plena aptitud intelectual, formando una personalidad real a fuerza de solidaridad y de conciencia de su misión, y que, recurriendo a toda fuente de cultura, brote de donde brotare, con tal que la linfa sea pura y diáfana, se propusiera adquirir los medios de nacionalizar la ciencia, de mexicanizar el saber (Sierra, 1910).

Para ello, asignó al maestro universitario una "divina misión", la cual consistía en formar sujetos morales, preocupados por la vida social. Así, le corresponde al maestro "saturar al hombre de espíritu de sacrificio, para hacerle sentir el valor inmenso de la vida social, para convertirlo en un ser moral en toda la belleza serena de la expresión" (Sierra, 1910).

\section{José Vasconcelos (1920-1921)}

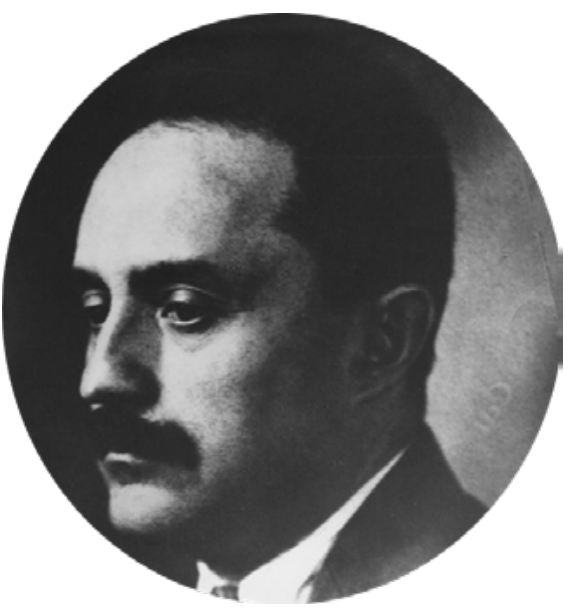

Al llegar a la rectoría de la Universidad, buscando poner en práctica los ideales de la Revolución Mexicana, Vasconcelos mencionó, en torno al tema que aquí nos congrega, lo siguiente: Los educadores de nuestra raza deben tener en cuenta que el fin capital de la educación es formar hombres capaces de bastarse a sí mismos y emplear su energía sobrante en el bien de los demás. Esto que teóricamente parece muy sencillo, es, sin embargo, una de las más difíciles empresas, una empresa que requiere verdadero fervor apostólico (Vasconcelos, 1920). 


\section{Ignacio García Téllez (1929-1932)}

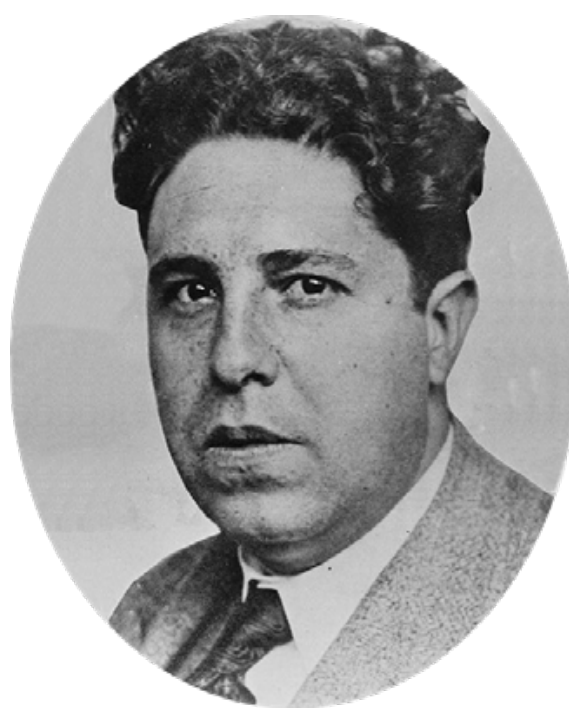

Por su parte, el primer rector de la ya autónoma Universidad Nacional de México, Ignacio García Téllez aludió a la importancia de revisar los planes de estudio que las Academias, es decir, los docentes y sus enseñanzas, llevaban a la práctica para formar universitarios que manejaran la teoría, pero que supieran aplicarla en su contexto:

Toca, en consecuencia, a los miembros honorables de este Consejo que hoy se instala y a las Academias que los asesoran, revisar los planes de estudios, haciendo que el alumno no se indigeste de enciclopedismo teórico, sino que complete su enseñanza con el conocimiento exacto de nuestro ambiente, con el contacto frecuente con los problemas que el campesino, el obrero, el industrial o el comerciante plantean constantemente dentro de nuestro medio, haciendo de cada estudiante un hombre útil para la sociedad, no un burócrata mendicante que, congestionando su espíritu con ideas extranjeras, sólo inculca principios irrealizables, predica falsas utopías y presta servicios a quienes en la defensa de sus intereses creados fomentan la lucha de clases, mantienen sin perfeccionar la técnica industrial y desean la prosperidad del monopolio capitalista sobre la explotación del esfuerzo humano (García, 1931).

\section{Roberto Medellín Ostos (1932-1933)}

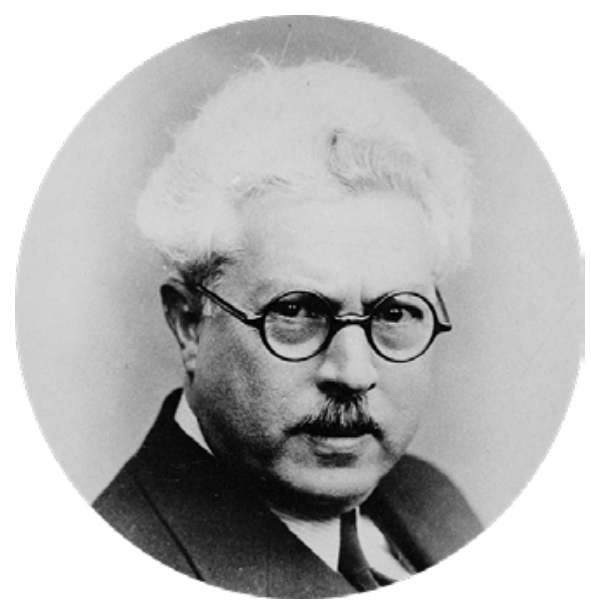

Concebía a la Universidad como la "máxima institución de cultura nacional" y señaló que la tarea a través de la cual la Universidad podía realizar su mejor obra social era la docencia:

Es necesario pensar que la mejor obra social que puede realizar la Universidad por medio de este programa es formar el espíritu de cuerpo de nuestra institución, para dar al país los hombres que, por sus conocimientos, por su moralidad y por su desprendimiento hagan la felicidad de México.

La única manera que tiene la Universidad de acercarse al pueblo es la de impartir una cultura amplia y profunda que, por el solo hecho de serlo, dará a los universitarios una actitud comprensiva de los problemas sociales y solidaria de las necesidades y aspiraciones de las grandes masas de la población (Medellín, 1932).

Para desarrollar esta tarea, Medellín Ostos indicó que se requería de docentes con ciertas características:

El verdadero maestro es el hombre que se prolonga a través del tiempo, en las juventudes, por su saber y por sus virtudes [...] [los maestros] son los únicos capaces de modelar el espíritu de los jóvenes [...]. Que el profesor no sea un mero repetidor de libros, que sea siempre ejemplo digno de imitar por los estudiantes, que sea un verdadero amigo de ellos, que piensa que la amistad no estriba en el halago, sino en la expresión clara y fiel de la verdad [...] 
Sé que hay muchos beneméritos maestros, y en el reconocerlo encuentro mi mayor satisfacción en estos momentos; pero quisiera que todos fueran como esos cuantos, y estuvieran dotados del mismo espíritu apostólico, del mismo desinterés por la enseñanza, de la misma dedicación al estudio y al trabajo (Medellín, 1932).

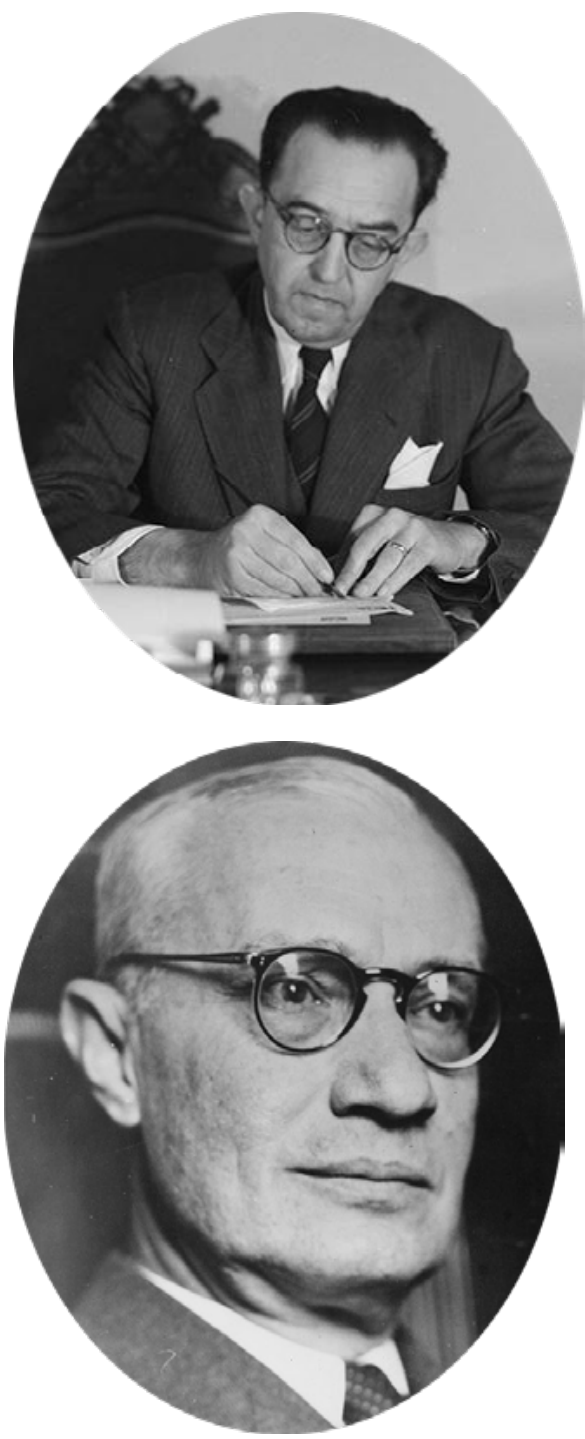

\section{Alfonso Caso Andrade (1944-1945)}

Alfonso Caso, durante su toma de protesta, buscó dejar en claro que lo que realicen las y los alumnos, así como las y los docentes de la institución será la forma en la que la sociedad mexicana vea a la Universidad: "La Universidad no es el rector, ni el Consejo, ni los directores, ni siquiera las Academias de Profesores y Alumnos, la Universidad Autónoma está integrada por sus estudiantes y sus maestros y será en la vida de México, lo que sean sus estudiantes y maestros" (Caso, 1944).

\section{Genaro Fernández MacGregor (1945-1946)}

Fernández MacGregor, alineándose al nuevo modelo de Universidad establecido por la recién aprobada Ley Orgánica de la UNAM (1945), indicó:

En nuestra Universidad, de glorioso abolengo, se ha formado la cultura que nos distingue, y que nos ha ayudado a cumplir nuestros destinos. En ella se modela el alma de la juventud mexicana que tendrá en sus manos, un poco más tarde, el buen nombre y la grandeza de México.

Magna obra ésta, que no puede verificarse sin la cooperación de todos: de los maestros, cuyas mentes deben estar abiertas a todas las corrientes del espíritu, y cuyos corazones deben impartir el resultado de sus estudios con la palabra viva que dictan el convencimiento y el amor (Fernández, 1945).

\section{Salvador Zubirán Anchondo (1946-1948)}

Durante el discurso pronunciado en la apertura del ciclo escolar del año 1947, citando al maestro Justo Sierra, indicó que la formación integral de los sujetos era la "misión más alta" de la Universidad Nacional:

Formar hombres es la más alta misión de la Universidad; pero hombres a quienes la cultura les dé la razón y el sentido de su tránsito por la vida, a quienes enseñe a constituirse en elementos útiles al bienestar social y colectivo. No permitir, como dijo un maestro insigne [Justo Sierra], "cultivar voluntades para cosechar egoísmos", sino por el contrario, saturar a los hombres de "espíritu de sacrificio y hacer- 
les reconocer el valor inmenso de la vida social"; que sean capaces de sentir los dolores de la humanidad, las angustias de su propio pueblo. Así, a nuestro Instituto no debe guiarlo tan sólo ese sentido de humana universalidad, sino también y muy principalmente, ese sentido humano pero vinculado a nuestra patria. Es preciso, como dijo el maestro Justo Sierra, mexicanizar el saber y grabar fuertemente en el estudiante universitario "la persuasión de que el interés en la ciencia y el interés en la patria, deben sumarse en el alma de todo estudiante mexicano" (Zubirán, 1947).

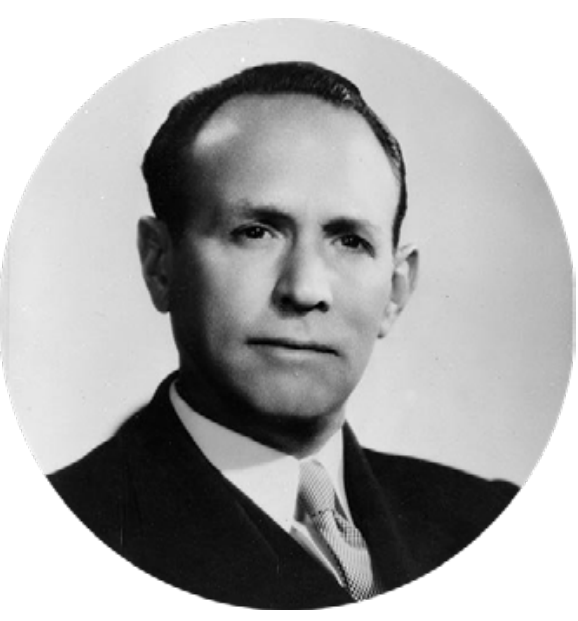

Respecto a la figura encargada de formar a los estudiantes universitarios, mencionó:

Hagamos ante todo que nuestros maestros universitarios sientan sobre sí la grave responsabilidad de constituirse en los mentores y guías de la juventud; que la educación universitaria conduzca a despertar las energías radicales del joven, a dirigir la promoción de sus sentimientos y de su voluntad, a crearle convicciones haciéndole intervenir activamente en la adquisición de los conocimientos y de los conceptos, y más que nada a modelar su carácter.

La labor del maestro no es sólo la de incrustar en el cerebro del alumno las cosas que ha de aprender y transformarlo en un receptáculo pasivo de ideas a las que él no tenga que añadir ninguna especie de elaboración; debe acostumbrársele desde temprano a luchar con las dificultades y no sólo hacerlo almacenar por un corto período de tiempo lo que se pretende enseñarle. Para ello, como afirma Giner de los Ríos, el maestro no debe mantener con el discípulo únicamente ese nexo débil y superficial, que deja a ambos extraños uno al otro y que origina que el alumno aprenda, pero sin interés ni amor a lo que estudia, sin idealizar su esfuerzo, formándose así disipado, frívolo, sordo a los dolores de la humanidad (Zubirán, 1947).

\section{Pablo González Casanova (1970-1972)}

González Casanova refiriéndose alaslabores que cada uno de los actoresuniversitarios, en este caso los docentes, debían desempeñar para propiciar el desarrollo del proyecto referente a la construcción de una nueva Universidad, mencionó:

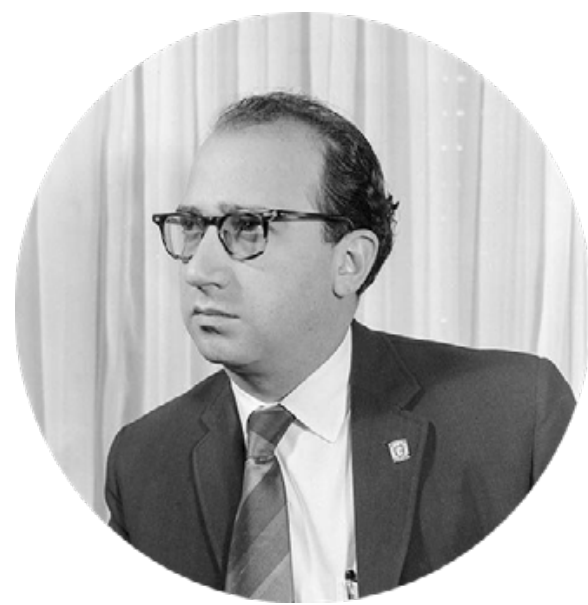

El verdadero profesor es aquel que sigue estudiando y el verdadero estudiante es aquel que también aprende a enseñar. [...] Los profesores no podemos limitarnos a ser profesores de especialidad sino de carácter, de serenidad, de conducta. No debemos tampoco limitarnos a dictar clase o a investigar: necesitamos proponernos, como diaria tarea, la construcción de pequeñas comunidades de diálogo de generaciones, en formas prácticas, viables, constantes, que pensamos estimular ampliamente en el futuro y para las que pediremos sus ideas, sus opiniones, sus reflexiones, a estudiantes y maestros (González, 1970). 


\section{Octavio Rivero Serrano (1981-1985)}

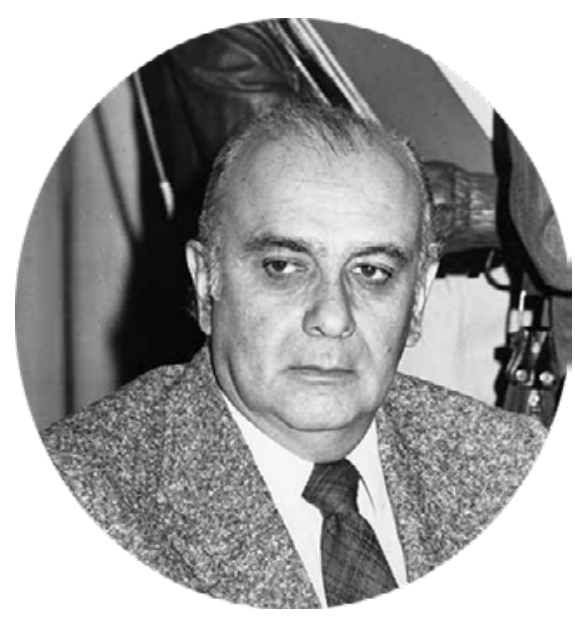

En su toma de protesta como rector, mencionó que la "misión fundamental" de las universidades, desde que éstas emergieron, ha sido la docencia y, en este sentido, la reafirmó como una actividad sustancial de la institución:

La misión fundamental de las universidades no se ha modificado: hoy más que nunca, les corresponde formar a los profesionistas, especialistas e investigadores que, altamente capacitados, se constituyan en los elementos de transformación económico-social del país en que viven.

Sólo así los universitarios serviremos al país; sólo así produciremos una movilidad social real que sea generadora de bienestar para las mayorías que son nuestra preocupación fundamental; sólo así, produciendo los recursos humanos comprometidos en vincular sus capacidades técnico-científicas con las necesidades del país y de nuestros compatriotas, mediante su trabajo, sus acciones, su capacidad de generar empleos y la elevación de los niveles de productividad, estaremos en la amplitud de influir en el desarrollo económico, social y cultural de México (Rivero, 1981).

\section{Jorge Carpizo MacGregor (1985-1989)}

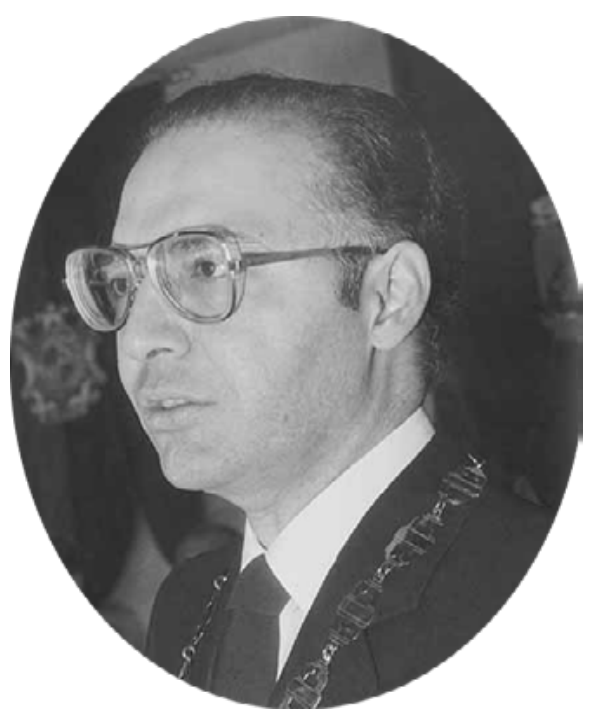

El día que tomó posesión como rector enfatizó que para un buen funcionamiento de la Universidad era necesario identificar la importancia de las tareas que debía realizar cada uno de los miembros de la comunidad universitaria, con el objetivo de que la institución mantuviera su alto nivel académico:

El nivel académico de una Universidad depende del nivel de sus profesores e investigadores. Los integrantes de este sector universitario comprendemos que necesitamos actualizarnos permanentemente, que necesitamos saber cómo enseñar mejor y ser estudiantes y estudiosos de por vida. El magisterio es una hermosa vocación de carácter social y debemos estar orgullosos de atender a ella: estamos ayudando a formar las mentes y las voluntades de quienes van a decidir el futuro de México (Carpizo, 1985).

Una vez mencionado lo anterior indicó que era necesario que la Universidad, a pesar de poseer ya un alto nivel académico, tomara medidas para la superación académica, puesto que:

La calidad de la Universidad se encuentra en relación directa con la de su personal académico.

Desde su fundación, la Universidad Nacional ha sido un centro de primordial importancia en la cultura mexicana. Relevancia y prestigio le ha otorgado su profesorado. La galería de nuestros maestros e investigadores coincide, en buena parte, con la de los hombres que han hecho la ciencia, la tecnología, las humanidades y las artes del México contemporáneo. Creadores de cosas nuevas 
y factores esenciales del porvenir, forjadores de hombres, nuestros profesores e investigadores han convertido a la Universidad en un polo de cultura que ejerce su influencia creadora, sin limitarse a su propio ámbito, sino antes bien, irradiándola sobre la nación entera. [...] Los autores de nuestra Ley Orgánica acertaron al definir al profesor al mismo tiempo como investigador, pues el trabajo académico es a la vez búsqueda y comunicación, o bien, diálogo que tiene como sustento la investigación. Así pues, en rigor, todo profesor debe investigar y todo investigador debe ejercer la docencia: ésta es la condición que subyace a nuestra legislación y un principio inherente al trabajo académico.

Al ejercer la docencia se transmite y recrea el conocimiento desde una posición personal determinada, en el marco de los planes y programas de estudios y bajo el principio de libertad de cátedra.

La recreación del conocimiento no es sólo el fruto de un intento de adecuación didáctica, sino también del diálogo con los estudiantes. De aquí que la docencia exija, ante todo, una actitud de probidad intelectual y de aprecio y respeto a los alumnos. El académico en funciones de docencia ha de trasmitir conocimientos al igual que valores e ideales (Carpizo, 1988).

\section{José Sarukhán Kermez (1989-1997)}

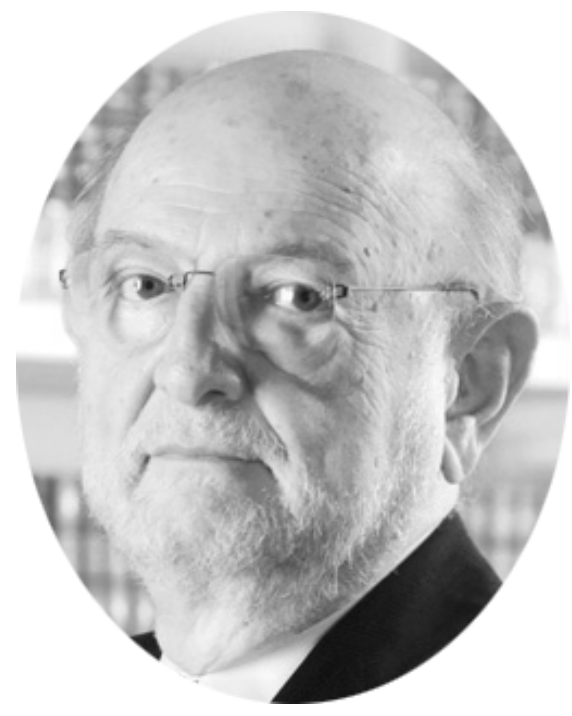

El rector Sarukhán Kermez mencionó que la UNAM era el "proyecto cultural" más importante en el México del siglo xx, e indicó que ello se debía, principalmente, al trabajo de docentes e investigadores:

Nuestra institución está enriquecida por la presencia y el trabajo creativo de varios cientos de profesores e investigadores que serían el orgullo de las mejores instituciones académicas del mundo. Su labor en la filosofía, en las artes, en las ciencias sociales, en las humanidades, en las ingenierías y en las ciencias exactas y naturales, es frecuente y repetidamente reconocida con premios y distinciones que halagarían a lo mejor del intelecto mundial. No exagero al decir que la comunidad académica de nuestra Universidad, como una parte muy importante del total del país, conforma uno de los sectores sociales que más han honrado y prestigiado el nombre de México en el ámbito internacional (Sarukhán, 1989).

A su vez indicó que su tarea central en la rectoría sería contribuir al proceso de "academizar" la Universidad puesto que la tarea académica es la que diferencia a la Universidad de otras organizaciones sociales. Este término de "academización" fue entendido como el proceso para realizar la generación de: "medidas concretas y adoptar las actitudes que pongan al personal académico y a la vida académica de la comunidad universitaria como el objetivo central de la institución" (Sarukhán, 1989):

El personal académico de la Universidad Nacional es su mayor riqueza y en él deberá apoyarse cualquier acción para lograr un mayor desarrollo académico. Esta riqueza de la Universidad también lo debe ser para el país, en su esfuerzo por superar las dificultades que en la actualidad nos afectan, y por alcanzar un futuro más propio que nos coloque como una nación más integrada en lo cultural, más independiente en lo económico y mucho más justa en lo social (Sarukhán, 1989). 


\section{Francisco Barnés de Castro (1997-1999)}

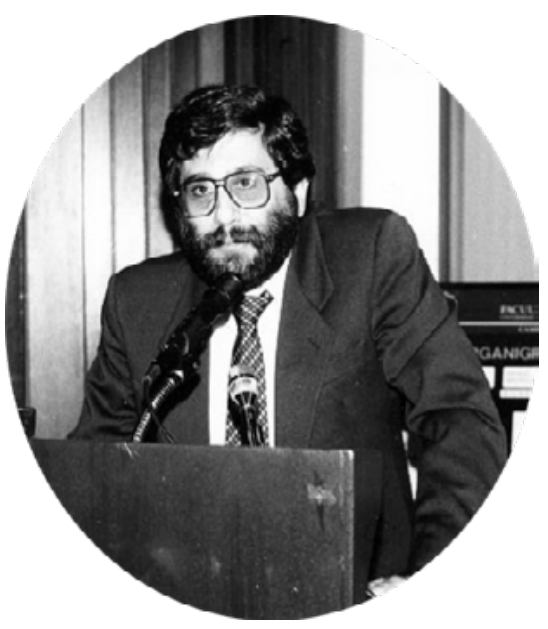

Al recibir el cargo como rector de la Universidad Nacional, el Dr. Barnés mencionó la serie de retos a los que se enfrentaría la institución, en gran medida con el cambio de milenio que se acercaba. Así, hizo explícita la tarea que le correspondería realizar a la Universidad:

A las universidades les corresponde jugar un papel determinante en este proceso de transformación, formando los recursos humanos altamente preparados que el país requiere, aportando conocimientos e información necesarios para una mejor toma de decisiones, tanto en el ámbito tecnológico como en el económico y social, y contribuyendo a preservar y enriquecer los valores de la cultura nacional (Barnés, 1997).

Haciendo referencia a la labor docente de la Universidad, menciona:

La misma labor educativa de las universidades sufrirá profundos cambios, al menos en lo que se refiere a la función tradicional de transmisión de conocimientos. La actividad docente deberá orientarse al desarrollo de capacidades y destrezas creativas, y a la habilidad para formular preguntas más pertinentes y encontrar mejores respuestas (Barnés, 1997).

\section{Juan Ramón de la Fuente (1999-2007)}

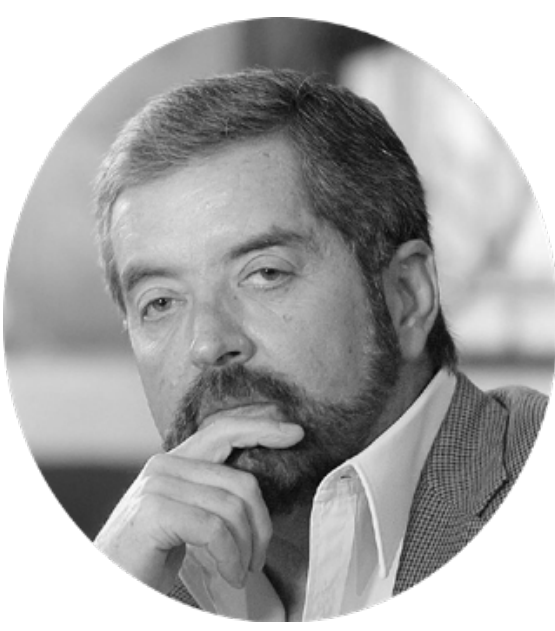

Aludiendo a los tiempos difíciles por los que atravesaba la Universidad, ${ }^{2}$ aunado al proceso mundial de "globalización y revolución tecnológica" que condicionan la educación superior, el rector buscó dar respuesta a la pregunta constante de estos tiempos: ¿cuál era el papel de las universidades dentro de ese escenario? Y para ello aludió a la importancia de revalorar a las y los docentes y su labor:

En este contexto, la universidad confronta el doble reto de mantenerse a la vanguardia de la tecnología educativa, y al mismo tiempo fortalecer los principios de rigor académicos, libertad de cátedra y compromiso social que le han dado sustento y razón de ser. [...] Al mismo tiempo hay que revalorar la función docente. Plantearse sin titubeos cómo debe entenderse el trabajo de enseñar, formar y educar, de cara a la globalización, a la sociedad del conocimiento (De la

${ }^{2}$ Al iniciar su tercer año de actividades (1999), el doctor Francisco Barnés de Castro propuso una reforma al Reglamento General de Pagos, hecho que marcaría su administración ya que no sería bien recibida por diversos sectores de la comunidad universitaria, razón por

la cual tuvo lugar un movimiento que derivó en una huelga estudiantil que duraría diez meses.
Así, menciona de manera concisa que educar, tarea de las maestras y maestros universitarios, es:

[...] mucho más que proporcionar información y transmitir contenidos epistemológicos. Educar es formar personalidades, construir a los sujetos éticos que habrán de asimilar y hacer suyo todo un orden cultural y moral en el cual los conocimientos adquiridos en la universidad mantengan pertinencia y sentido. Educar es 
forjar seres humanos sensibles, autónomos, críticos y creativos, comprometidos con la comunidad que pertenecen, aptos para el ejercicio consciente de la democracia, así como para enriquecer y dar continuidad a la tradición cultural en la cual están inmersos (De la Fuente, 2001).

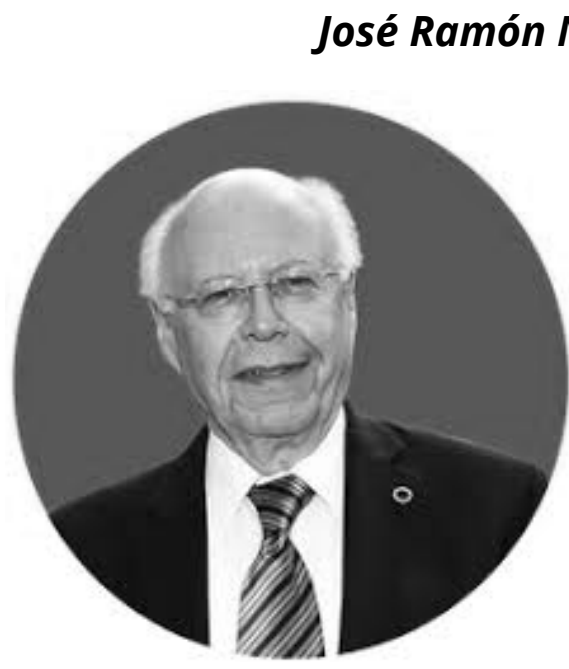

Por su parte, el doctor José Narro, en el discurso pronunciado en la toma de posesión del cargo denotó los alcances de la actividad docente, que como ya se ha reiterado, no se restringe únicamente a la formación de profesionales:

En sus programas e instalaciones se forman profesionales capaces y con conciencia. A través del trabajo de sus académicos, el conocimiento no sólo se transmite a las nuevas generaciones, se genera y aplica a la solución de problemas que aquejan a la sociedad. En la Universidad se busca transformar el saber y el quehacer de académicos y estudiantes, en servicios para la colectividad, en extensión del conocimiento, en nuevas modalidades de difusión de la cultura (Narro, 2007).

De manera específica, el Dr. José Narro, en un mensaje emitido a los docentes el Día del Maestro del año 2015, les dice:

Ustedes tienen un papel insustituible en la educación, en ese bien social liberador de los potenciales del ser humano. De su tarea depende en parte el desarrollo de la inteligencia y la creatividad de sus alumnos. Con su trabajo contribuyen a mejorar el bienestar y la dignidad del estudiante y familias. Ustedes son escultores de ciudadanos informados y con principios (Narro, 2015).

Haciendo alusión también a la importancia de la formación continua del docente y el hecho de que la tarea docente no se restringe al espacio del aula, reconoce a:

Aquellos académicos generosos que comparten sus conocimientos y que se esfuerzan por estar actualizados. De los que enseñan y educan como parte de un estilo de vida. De quienes lo hacen en el aula y fuera de ella, en los auditorios y los corredores, en los cubículos y en los laboratorios o talleres (Narro, 2015).

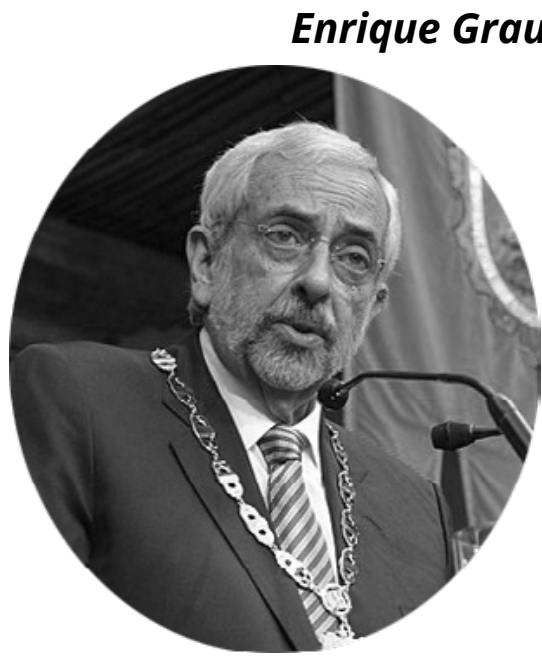

En su discurso de toma de posesión del cargo, el rector actual de la unAm resaltó la labor de educar como la principal misión de la Universidad, aludiendo a la trascendencia que ésta tiene en la sociedad a través de sus estudiantes y egresados universitarios:

Educar es nuestra principal misión. Educar sin distingo de ideologías, preferencias o condiciones socioeconómicas. Formar a todos aquellos jóvenes que por sus características académicas se hayan ganado el derecho de ser universitarios. Y nuestra obligación es hacerlo en las mejores condiciones y egresar a nuestros estudiantes competentes y capaces de reformar a la sociedad. Queremos también egresados reflexivos, creativos, innovadores, con compromiso social e inquebrantables en su ética profesional (Graue, 2015). 


\section{Consideraciones finales}

Este artículo busca destacar a través de los discursos de los rectores que, aún con muchos retos por atender, la UNAM ha sabido afrontar los cambios que la realidad le ha planteado, y que el respeto y exaltación a la labor que las maestras y maestros desempeñan ha sido una constante. Lo anterior deja en claro que el hecho de que se posicione como la mejor del país y de América Latina se debe en gran medida a la tarea que desarrollan día con día sus docentes en los diversos espacios universitarios.

A través de sus discursos, los rectores hacen explícito que el ser docente unamita es un gran privilegio, el cual conlleva una gran responsabilidad, ya que ser maestra o maestro va más allá de la vocación, implica un compromiso profundo con la actividad docente, con la institución, con las y los estudiantes, y consigo mismo. Supone, a su vez, una formación continua en materia pedagógica, una actualización permanente en la disciplina que se enseña, un interés constante en el aprendizaje de las y los estudiantes, así como sumergirse en la vida colegiada.

Por otra parte, es fundamental que la institución provea al profesorado de los medios suficientes para que pueda ejecutar su labor: infraestructura, recursos y materiales, y una propuesta formativa permanente, entre otros aspectos.

A modo de cierre, considero oportuno mencionar que la docencia es una de las actividades más gratificantes que existen, los beneficios que esta tarea deja, tanto en el plano intelectual como sentimental, son incomparables. Cada docente es único y forja en sus estudiantes conocimientos y actitudes distintas, porque las maestras y los maestros también enseñan debido a y de acuerdo con lo que son como seres humanos.

\section{Referencias}

- Bain, Ken (2007). Lo que hacen los mejores profesores de universidad (2da. ed., Óscar Barbéra [Trad.]). Valencia: Universitat de València.

* Imbernon, Francesc (2009). Mejorar la enseñanza y el aprendizaje en la universidad (Cuadernos de docencia universitaria 14). Barcelona: ICE Universitat de BarcelonaOctaedro. Recuperado de: http://www.ub.edu/ice/sites/default/files//docs/ qdu/14cuaderno.pdf.

Solís Quiroga, Héctor (1961). El ser y el deber ser de la Universidad de México. México: Asociación Mexicana de Sociología. 


\section{Discursos}

* Barnés de Castro, Francisco (2014). Toma de posesión de Francisco Barnés de Castro el 6 de enero de 1997. En José Gallegos Téllez (Ed.), Discursos de toma de posesión de los rectores de la Universidad Nacional Autónoma de México 19102011. México: IISUE-UNAM. Recuperado de: http://132.248.192.241/ editorial/wpcontent/uploads/2014/10/Discursos-de-rectores-UNAM1.pdf.

* Carpizo MacGregor, Jorge (1999). El ser y el deber ser de la Universidad Nacional Autónoma de México. En Xavier Cortés Rocha y Adolfo Rodríguez Gallardo (Coords.), Visión de la Universidad. Una visión plural. México: UNAM (enunciado en 1988).

- Carpizo MacGregor, Jorge (2014). Toma de posesión de Jorge Carpizo MacGregor el 2 de enero de 1985. En José Gallegos Téllez (Ed.), Discursos de toma de posesión de los rectores de la Universidad Nacional Autónoma de México 1910-2011. México: IISUE-UNAM. Recuperado de: http://132.248.192.241/ editorial/wp-content/ uploads/2014/10/Discursos-de-rectores-UNAM1.pdf.

* Caso Andrade, Alfonso (2014). Discurso pronunciado el 15 de agosto de 1944. En José Gallegos Téllez (Ed.), Discursos de toma de posesión de los rectores de la Universidad Nacional Autónoma de México 1910-2011. México: IISUE-UNAM. Recuperado de: http://132.248.192.241/ editorial/wp-content/uploads/2014/10/ Discursos-de-rectores-UNAM1.pdf.

* De la Fuente, Juan Ramón (2001, marzo-mayo). Perspectivas de la educación superior en México. En Revista de la Universidad de México, núm. 602-604. México: UNAM. Recuperado de: http://www.revistadelauniversidad.unam.mx/ojs rum/ files/journals/1/articles/15172/public/15172-20570-1-PB.pdf.

* Fernández MacGregor, Genaro (2014). Discurso pronunciado el 24 de marzo de 1945. En José Gallegos Téllez (Ed.), Discursos de toma de posesión de los rectores de la Universidad Nacional Autónoma de México 1910-2011. México: IISUE-UNAM. Recuperado de: http://132.248.192.241/ editorial/wp-content/uploads/2014/10/ Discursos-de-rectores-UNAM1.pdf.

* García Téllez, Ignacio (1999). Palabras del rector Ignacio García Téllez al Primer Consejo Universitario el 31 de julio de 1929. En Xavier Cortés Rocha y Adolfo Rodríguez Gallardo (Coords.), Visión de la Universidad. Una visión plural. México: UNAM.

* González Casanova, Pablo (15 de mayo de 1970). Discurso del Doctor Pablo González Casanova. En Gaceta UNAM, vol. xIX, núm. 5, México: UNAM. Recuperado de: http://acervo.gaceta.unam.mx/index.php/gum70/issue/view/651/showToc.

* Medellín Ostos, Roberto (1999). Discurso pronunciado por el nuevo rector de la Universidad, químico Roberto Medellín, en el acto de protesta de su cargo el 12 de septiembre de 1932. En Xavier Cortés Rocha y Adolfo Rodríguez Gallardo (Coords.), Visión de la Universidad. Una visión plural. México: UNAM. 
* Narro Robles, José (2007). Toma de posesión de José Narro Robles el 20 de noviembre de 2007. En José Gallegos Téllez (Ed.), Discursos de toma de posesión de los rectores de la Universidad Nacional Autónoma de México 1910-2011. México: IISUE-UNAM. Recuperado de: http://132.248.192.241/ editorial/wp-content/ uploads/2014/10/Discursos-de-rectores-UNAM1.pdf.

* Narro Robles, José (2015). Mensaje del Rector en el Día del Maestro. En Gaceta UNAM, núm. 4694, México: UnAM. Recuperado de: http://www.gaceta.unam. $\mathrm{m} \times / 20150518 /$.

* Rivero Serrano, Octavio (2014). Toma de posesión de Octavio Rivero Serrano el 3 de enero de 1981. En José Gallegos Téllez (Ed.), Discursos de toma de posesión de los rectores de la Universidad Nacional Autónoma de México 1910-2011. México: IISUE-UNAM. Recuperado de: http://132.248.192.241/ editorial/wp-content/ uploads/2014/10/Discursos-de-rectores-UNAM1.pdf.

* Sarukhán Kermez, José (2014). Discurso pronunciado el 2 de enero de 1989. En José Gallegos Téllez (Ed.), Discursos de toma de posesión de los rectores de la Universidad Nacional Autónoma de México 1910-2011. México: IISUE-UNAM. Recuperado de: ht tp://132.248.192.241/ editorial/wp-content/uploads/2014/10/Discursos-derectores-UNAM1.pdf.

* Sierra, Justo (2004). Discurso inaugural de la Universidad Nacional (Colección: Pequeños Grandes Ensayos). México: UnAm (enunciado en 1910).

* Sierra, Justo (1984). La Universidad Nacional. Proyecto de creación. En Obras Completas. La educación Nacional (3a ed., t. VIII). México: UNAM (original de 1881).

* Vasconcelos, José (2014). Declaraciones del señor licenciado don José Vasconcelos, con motivo de la toma de posesión del cargo de rector de la Universidad Nacional de México. En José Gallegos Téllez (Ed.), Discursos de toma de posesión de los rectores de la Universidad Nacional Autónoma de México 1910-2011. México: IISUEUNAM (enunciado en 1920). Recuperado de: http://132.248.192.241/ editorial/wpcontent/uploads/2014/10/Discursos-de-rectores-UNAM1.pdf.

- Zubirán Anchondo, Salvador (1999). Discurso del rector doctor Salvador Zubirán Anchondo, durante la ceremonia de apertura de cursos en el Palacio de Bellas Artes, febrero de 1947. En Xavier Cortés Rocha y Adolfo Rodríguez Gallardo (Coords.), Visión de la Universidad. Una visión plural. México: UNAM.

* Normatividad UNAm (2003). Marco Institucional de Docencia. México: UnAm. Recuperado de: www.abogadogeneral.unam.mx/legislacion/abogen/documento. html?doc id=44. 


\section{Imágenes de los rectores}

[Ordenadas de acuerdo con el período del rector].

- IISUe-AHUnAm (s.f.). [Justo Sierra] [imagen]. Recuperada de: http://www.ahunam. unam.mx/images/albums/4.18/album/017\%20Reproducciones\%20y\%20 fotografias\%20sobre\%20temas\%20universitarios/index.html\#RED-06430.jpg.

- IISUe-Ahunam (s.f.). [José Vasconcelos] [fotografía]. Recuperada de: http:// www.ahunam.unam.mx/images/albums/2.11/album/01\%20Rectores/index. html\#CU-000031.jpg.

* IISUe-Ahunam (s.f.). [Ignacio García Téllez] [fotografía]. Recuperada de: http:// www.ahunam.unam.mx/images/albums/2.11/album/01\%20Rectores/index. html\#CU-000072-00.jpg.

* IISUe-Ahunam (s.f.). [Roberto Medellín Ostos] [fotografía]. Recuperada de: http:// www.ahunam.unam.mx/images/albums/2.11/album/01\%20Rectores/index. html\#CU-000076-03.jpg.

- IISUe-Ahunam (s.f.). [Alfonso Caso Andrade] [fotografía]. Recuperada de: http:// www.ahunam.unam.mx/images/albums/4.18/album/008\%20Alfonso\%20 Caso\%20y\%20Andrade/index.htm|\#RED-00052.jpg.

* IISUE-AHUNAM (s.f.). [Genaro Fernández MacGregor] [fotografía]. Recuperada de: http://www.ahunam.unam.mx/images/albums/2.11/album/01\%20Rectores/ index.html\#CU-000151.jpg.

* IISUE-AHUNAM (S.f.). [Salvador Zubirán Anchondo] [fotografía]. Recuperada de: http:// www.ahunam.unam.mx/images/albums/4.18/album/010\%20\%20Salvador\%20 Zubiran\%20Anchondo/index.html\#RED-00070-00.jpg.

* IISUe-Ahunam (s.f.). [Pablo González Casanova] [fotografía]. Recuperada de: http://www.ahunam.unam.mx/images/albums/4.18/album/015\%20Pablo\%20 Gonzanez\%20Casanova/index.htm|\#RED-05467.jpg.

* IISUe-Ahunam (s.f.). [Octavio Rivero Serrano] [fotografía]. Recuperada de: http:// www.ahunam.unam.mx/images/albums/2.11/album/07\%200ctavio\%20 Rivero\%20Serrano/index.htm|\#CU-002618-00.jpg.

* IISUe-Ahunam (s.f.). [Jorge Carpizo MacGregor] [fotografía]. Recuperada de: http://www.ahunam.unam.mx/images/albums/2.11/album/45\%20Jorge\%20 Carpizo\%20M/SECC\%20JCM\%20Retratos/1985/index.html\#CU-009988.jpg.

* El Colegio Nacional (s.f.). José Sarukhán [fotografía]. Recuperada de: http://colnal. $\mathrm{mx} / \mathrm{members/jose-sarukhan.}$

- Patronato de la Facultad de Química (s.f.). Exrector Dr. Francisco Barnés de Castro Director de la Facultad de Química a lo largo de la 1er. Campaña [fotografía]. Recuperada de: https://patronatofa.org.mx/campanas/origenes/.

* Eneas De Troya (18 mayo, 2012). Juan Ramón de la Fuente 2012 Cropped [fotografía]. Recuperada de: https://commons.wikimedia.org/w/index.php?curid=37144075. 
* Gaceta Facultad de Medicina (25 mayo, 2018). Doctor José Narro Robles [fotografía]. Recuperada de: http://gaceta.facmed.unam.mx/index.php/2018/05/25/ritmoy-rumbo-de-la-salud-en-mexico-conversaciones-con-los-secretarios-desalud-1982-2018/

- dgCS UNAM (18 nov, 2015). Enrique Graue Wiechers. Recuperada de: https:// commons. wikimedia.org/w/index.php?curid=62043915.

\section{Cómo citar este artículo}

* Nieto Juárez, Tania Itzel (2019). Docencia universitaria desde la perspectiva de los rectores de la UnAM. Revista Digital Universitaria (RDU). Vol. 20, núm. 3 mayo-junio. DOI: http://doi.org/10.22201/codeic.16076079e.2019.v20n3.a10.

Recepción: 27/02/19. Aprobación: 09/04/19 